\title{
Role of the (Mn)superoxide dismutase of Enterococcus faecalis in the in vitro interaction with microglia
}

\author{
Samuele Peppoloni, ${ }^{1}$ Brunella Posteraro, ${ }^{2}$ Bruna Colombari, ${ }^{1}$ \\ Lidia Manca, ${ }^{1}$ Axel Hartke, ${ }^{3}$ Jean-Christophe Giard, ${ }^{3}$ \\ Maurizio Sanguinetti, ${ }^{2}$ Giovanni Fadda ${ }^{2}$ and Elisabetta Blasi ${ }^{4}$ \\ ${ }^{1}$ Dipartimento di Scienze di Sanità Pubblica, Università degli Studi di Modena e Reggio Emilia, \\ Via Campi 287, 41125 Modena, Italy \\ ${ }^{2}$ Institute of Microbiology, Catholic University of the Sacred Heart, 00168 Rome, Italy \\ ${ }^{3}$ Laboratoire de Microbiologie de l'Environnement, EA 956 USC INRA, IRBA, \\ Université de Caen, 14032 Caen Cedex, France \\ ${ }^{4}$ Dipartimento ad Attività Integrata di Laboratori, Anatomia Patologica e Medicina Legale, \\ Azienda Ospedaliero-Universitaria di Modena, Via del Pozzo no 71, 41124 Modena, Italy
}

Correspondence

Samuele Peppoloni

samuele.peppoloni@unimore.it

Received 3 December 2010

Revised 10 March 2011

Accepted 4 April 2011

\begin{abstract}
Enterococcus faecalis is a significant human pathogen worldwide and is responsible for severe nosocomial and community-acquired infections. Although enterococcal meningitis is rare, mortality is considerable, reaching $21 \%$. Nevertheless, the pathogenetic mechanisms of this infection remain poorly understood, even though the ability of $E$. faecalis to avoid or survive phagocytic attack in vivo may be very important during the infection process. We previously showed that the manganese-cofactored superoxide dismutase (MnSOD) SodA of E. faecalis was implicated in oxidative stress responses and, interestingly, in the survival within mouse peritoneal macrophages using an in vivo-in vitro infection model. In the present study, we investigated the role of $\mathrm{MnSOD}$ in the interaction of $E$. faecalis with microglia, the brain-resident macrophages. By using an in vitro infection model, murine microglial cells were challenged in parallel with the wildtype strain $\mathrm{JH} 2-2$ and its isogenic sodA deletion mutant. While both strains were phagocytosed by microglia efficiently and to a similar extent, the $\Delta \operatorname{sod} A$ mutant was found to be significantly more susceptible to microglial killing than $\mathrm{JH}_{2}-2$, as assessed by the antimicrobial protection assay. In addition, a significantly higher percentage of acidic $\Delta$ sodA-containing phagosomes was found and these also underwent enhanced maturation as determined by the expression of endolysosomal markers. In conclusion, these results show that the MnSOD of $E$. faecalis contributes to survival of the bacterium in microglial cells by influencing their antimicrobial activity, and this could even be important for intracellular killing in neutrophils and thus for $E$. faecalis pathogenesis.
\end{abstract}

\section{INTRODUCTION}

Enterococcus faecalis is a natural member of the digestive microflora in humans and many other animals but in some instances it becomes a pathogen capable of causing severe human diseases (Gilmore et al., 2002), such as bacteraemia (Suppli et al., 2010), endocarditis (Fernández Guerrero et al., 2007), neonatal sepsis (Sarkar et al., 2006) and meningitis (Pintado et al., 2003). Although enterococcal meningitis is rare, mortality is relatively high and occurs in $21 \%$ of cases, posing additional challenges for the

Abbreviations: DAPI, 4,6-diamidino-2-phenylindole; MnSOD, manganese-cofactored superoxide dismutase; ROS, reactive oxygen species. treatment of this infection (Pintado et al., 2003). In addition, the emergence of vancomycin- and multidrugresistant enterococci as important nosocomial pathogens is a cause of concern, as they are very difficult to control once established (Murray, 2000; Willems \& Bonten, 2007; Huycke et al., 1998).

Microglia are brain macrophages that share many, if not all, the properties of macrophages in other tissues including the generation of free radicals, such as reactive oxygen species (ROS) during the respiratory burst, which is regarded as an important defence mechanism of the central nervous system against intracellular micro-organisms (Rock et al., 2004). ROS formed during phagocytosis are 
primarily inside the phagolysosome, even though stimuli other than phagosome formation can induce ROS production in intracellular compartments (Bylund et al., 2010). E. faecalis has developed several enzymic and nonenzymic mechanisms to counteract deleterious effects of superoxide anion $\left(\mathrm{O}_{2}^{-\cdot}\right)$, hydrogen peroxide $\left(\mathrm{H}_{2} \mathrm{O}_{2}\right)$ and hydroxyl radical $\left(\mathrm{OH}^{\circ}\right)$, which range from DNA strand damage to peroxidation of membrane lipids (Imlay, 2003). An essential element of the primary antioxidant defence system of $E$. faecalis is the manganese-cofactored superoxide dismutase (MnSOD) SodA encoded by the sodA gene (Verneuil et al., 2006). In particular, we showed that the $E$. faecalis sodA deletion mutant was more sensitive to treatment with $\mathrm{H}_{2} \mathrm{O}_{2}$ than the isogenic parental strain JH2-2, whereas complementation of the $\Delta$ sodA mutant restored resistance to this oxidant. Furthermore, the $\Delta s o d A$ mutant exhibited decreased resistance to intracellular killing by murine peritoneum-resident macrophages compared with its parent $\mathrm{JH} 2-2$, suggesting that MnSOD may be considered to be a virulence factor (Verneuil et al., 2006).

As a consequence of renewed interest in microglia, insights are being gained from in vivo and in vitro studies on their physiological and pathological properties, supporting the defensive role of microglia against CNS infections (Rock et al., 2004). Thus, in-depth studies on the interaction of microglial cells with $E$. faecalis would greatly facilitate a better understanding of bacterial neuropathogenesis. In particular, the initial E. faecalis-to-microglia recognition phase as well as the post-phagocytosis intracellular events should be carefully investigated, since both steps seem to be relevant in the outcome of such pathogen-host cell interplay.

In this study, the JH2-2 wild-type strain and its $\Delta s o d A$ mutant were compared for the ability to interact with microglia. Moreover, the intracellular fate of the $\Delta \operatorname{sod} A$ mutant was examined in comparison with the wild-type strain in order to evaluate whether MnSOD might influence phagosome maturation following E. faecalis internalization by microglia.

\section{METHODS}

Microglial cells. The murine microglial cell line BV2, established as described previously (Blasi et al., 1990), was maintained in RPMI 1640 medium (Gibco) supplemented with $10 \%$ (v/v) fetal calf serum (heat-inactivated for $30 \mathrm{~min}$ at $56{ }^{\circ} \mathrm{C}$, EuroClone), gentamicin $(50 \mu \mathrm{g}$ $\mathrm{ml}^{-1}$; Bio Whittaker) and L-glutamine ( $2 \mathrm{mM}$; EuroClone), hereafter referred to as complete $\mathrm{RPMI}$, at $37{ }^{\circ} \mathrm{C}$ in a $5 \% \mathrm{CO}_{2}$ in air atmosphere. Then, BV2 cells were detached by vigorous shaking biweekly and fresh cultures were started at a concentration of $5 \times 10^{5}$ $\mathrm{ml}^{-1}$ the day before the experiment.

Bacterial strains, growth conditions and labelling procedure. $E$. faecalis JH2-2 wild-type and its derivatives, the $\Delta$ sodA mutant and a complemented $\Delta$ sodA strain (Verneuil et al., 2006), were used in this study. Briefly, the $\Delta$ sodA mutant was constructed by replacement of the wild-type gene with a mutated copy of $\operatorname{sod} A$ containing stop codons and a central deletion, whereas the complemented strain was obtained by knocking in the wild-type sodA allele into the $\Delta \operatorname{sodA}$ mutant (Verneuil et al., 2006). Bacteria were cultivated in brain heart infusion (BHI) medium (Oxoid) at $37^{\circ} \mathrm{C}$ with vigorous shaking (150 r.p.m.) to reach $\mathrm{OD}_{620} 0.2$, harvested by centrifugation and suspended at the desired concentration. For all fluorescence-based assays, $2 \times 10^{8}$ bacterial cells $\mathrm{ml}^{-1}$ were labelled with 4,6-diamidino2-phenylindole (DAPI) (Molecular Probes/Invitrogen; $1 \mu \mathrm{g} \mathrm{ml}^{-1}$ ). After incubation at $37{ }^{\circ} \mathrm{C}$ for $30 \mathrm{~min}$, bacteria were washed three times with PBS to remove unbound dye, and the bacteria were diluted to the desired concentration in complete RPMI medium without antibiotics. MICs of gentamicin and vancomycin were determined by a standard broth microdilution method (CLSI, 2010).

Microglia survival assay. Bacterial microglia survival was quantified utilizing a modified antibiotic-protection assay (Brett et al., 2008). Briefly, BV2 cells $\left(1 \times 10^{6} \mathrm{ml}^{-1}\right)$ were incubated for $1.5 \mathrm{~h}$ with each of the bacterial strains in complete RPMI medium without antibiotics at a host: pathogen ratio of $1: 5$. Cells were washed twice with PBS to remove extracellular bacteria and exposed for $2 \mathrm{~h}$ to gentamicin $\left(150 \mu \mathrm{g} \mathrm{ml}^{-1}\right)$ and vancomycin $\left(10 \mu \mathrm{g} \mathrm{ml}^{-1}\right)$ in fresh complete RPMI to suppress the growth of residual extracellular bacteria. BV2 cells were then washed twice with PBS and suspended in complete RPMI without antibiotics (time 0 ). At $0,2,4$ and $8 \mathrm{~h}$ post-infection, cells were lysed with $0.2 \%(\mathrm{v} / \mathrm{v})$ Triton $\mathrm{X}-100$ for $15 \mathrm{~s}$ to release intracellular bacteria, and serial dilutions of the lysates were plated onto BHI agar. The plates were incubated at $37{ }^{\circ} \mathrm{C}$ for $48 \mathrm{~h}$ and the c.f.u. counts were then used to enumerate bacterial loads. Control experiments were carried out to verify that the Triton X-100 treatment was not toxic for bacteria (data not shown).

Phagocytosis assay. To strengthen attachment of BV2 cells to the wells, Lab-Tek II chamber slides (Nalge Nunc International) were pretreated with poly-L-lysine (Sigma; $10 \mu \mathrm{g}$ per well) for $30 \mathrm{~min}$, and washed twice with PBS. BV2 cells $\left(2 \times 10^{6} \mathrm{ml}^{-1}\right.$ in complete RPMI, $100 \mu \mathrm{l}$ per well) were seeded, incubated as described for $24 \mathrm{~h}$ and then infected with $100 \mu \mathrm{l}$ of a suspension of $10^{7}$ DAPI-labelled bacteria $\mathrm{ml}^{-1}$ (see above) in complete RPMI (host: pathogen ratio of $1: 5$ ). After incubation for 1.5 or $3 \mathrm{~h}$, cells were then treated with trypan blue for $5 \mathrm{~min}$ to quench the fluorescence of the bacteria which were bound but not internalized by the microglia, washed three times in PBS to remove extracellular bacteria and fixed for 30 min with $4 \%$ paraformaldehyde (PFA) (Sigma) in PBS. The remaining fluorescence of the phagocytosed bacteria was visualized by epifluorescence microscopy. At least 200 microglial cells from each sample were examined and the percentage of cells that had intracellular bacteria was estimated as the ratio of the number of BV2 cells containing one or more bacteria to the total number of BV2 cells examined.

Phagosome acidification assay. Visualization of the acidic bacteria-containing phagosomes was performed as described previously (Orsi et al., 2009). Briefly, Lab-Tek II chamber slides were prepared and BV2 cells were infected as in the phagocytic assay. Then, the cells were washed to eliminate extracellular bacteria, and were exposed to $4 \mu \mathrm{l}$ of the acidotropic dye LysoTracker Red DND-99 (Molecular Probes/Invitrogen) at a final concentration of $5 \mu \mathrm{M} \mathrm{ml}^{-1}$. Thirty minutes before the end of incubation, an additional volume $(4 \mu \mathrm{l})$ of the same dye was added. Finally, $5 \mathrm{~min}$ before the end of incubation, $100 \mu \mathrm{l}$ trypan blue was dispensed into each well. After PFA fixing, cells with acidic phagolysosomes were washed and immediately visualized by epifluorescence microscopy. Successful acidification of phagosomes containing DAPI-labelled bacteria was indicated by the appearance of both LysoTracker Red DND-99 (red) and DAPI (blue) fluorescence within the phagosomes, so that merging of images of the different fluorescence channels resulted in purple fluorescence. For quantitative analysis, the percentage of acidic 
(purple) phagosomes per image was determined by counting purple phagosomes within the phagocytic cells.

Colocalization studies. Phagosome-maturation markers (Rab5, Rab7 and Rab9) and lysosome-associated membrane protein 2 (LAMP2) (Kinchen \& Ravichandran, 2008) were assessed in the colocalization studies. Briefly, BV2 cells were prepared as in the phagocytosis assay and allowed to take up DAPI-labelled bacteria. At 30 and 45 min post-infection, cells were washed, fixed with $4 \%$ (v/v) paraformaldehyde in PBS for $30 \mathrm{~min}$ and permeabilized for $10 \mathrm{~min}$ with $0.2 \%$ Triton X-100 in PBS. Cells were then incubated $(1 \mathrm{~h}$ at room temperature) with the primary antibodies (anti-Rab5, -Rab7, Rab9 or -LAMP2; Sigma), washed twice with PBS, and incubated (1 h at room temperature) with the secondary Chromeo 642 goat antirabbit IgG antibody (Active Motif). The overlay signals of DAPIlabelled bacteria (blue fluorescence) with the red fluorescence of secondary antibody Chromeo 642 were interpreted as colocalization by using epifluorescence microscopy. Association of DAPI with Rab5, Rab7-, Rab9- or LAMP2-positive vacuoles was quantified by scoring the colocalization phenotypes of at least 100 individual bacteria from each well in the chamber slides.

Epifluorescence microscopy. Prior to visualization, Lab-Tek II chamber slides were washed with PBS and then treated with prolong gold antifade reagent (Invitrogen) to suppress photo-bleaching and preserve the fluorescence signal. Epifluorescence and differential interference contrast microscopy were performed with a Nikon Eclipse 90i imaging system equipped with Nomarski DIC optics (Nikon Instruments). At each time point, samples were photographed with a DS-2Mv Nikon digital camera, and the resulting photographs were analysed by using the Nikon NIS-ELEMENTS version D3.1 software.

Statistical analysis. Results are reported as mean $\pm S D$ of three independent experiments and triplicates were prepared for each time point and experiment. In all epifluorescence studies at least 200 cells per group were scored. To evaluate the statistical significance of the results, Student's unpaired $t$-test or analysis of variance (one-way ANOVA) with a Bonferroni correction post-test was performed using JMP software. $P$-values $<0.05$ were considered significant.

\section{RESULTS}

In the present study, E. faecalis wild-type strain $\mathrm{JH} 2-2$, its $\Delta \operatorname{sod} A$ mutant and the complemented $\Delta \operatorname{sod} A$ mutant were used to investigate the role of MnSOD in the interaction between $E$. faecalis and microglia. Upon infection with each strain, microglial cells were assessed for phagocytosis, antimicrobial activity and phagosome maturation.

\section{MnSOD does not influence the susceptibility of E. faecalis to phagocytosis by microglia}

We performed fluorescence studies to characterize the interaction of the $\Delta$ sodA mutant with BV2 murine microglia. Previously, we had established a quantitative phagocytosis assay, in which the fluorescence only from attached bacteria, and not from those internalized by microglial cells, was quenched by trypan blue (Peppoloni et al., 2010). Thus, we could reliably detect internalized enterococci alone and perform time-course studies of the phagocytic activity of the BV2 cells against JH2-2, $\Delta \operatorname{sodA}$ mutant and the complemented $\Delta$ sodA strain. Fig. 1 depicts the number of phagocytic cells that ingested one or more bacteria at 1.5 and $3 \mathrm{~h}$ post-infection. The phagocytic uptake of the three strains followed a similar pattern with time, as the percentages of BV2 cells with internalized JH22, $\Delta$ sodA mutant or the complemented $\Delta$ sodA strain were slightly, but not statistically significantly, different. These data show that MnSOD does not affect the phagocytic activity of murine microglia against $E$. faecalis.

\section{MnSOD affects $E$. faecalis survival within microglia}

To examine the ability of the $\Delta \operatorname{sod} A$ mutant to survive within BV2 cells, a gentamicin/vancomycin protection assay was performed. BV2 cells were infected with $\mathrm{JH} 2-2$, the $\Delta$ sodA mutant or the complemented $\Delta$ sodA strain for $1.5 \mathrm{~h}$ and bacterial loads were determined at $0,2,4$ and $8 \mathrm{~h}$ post-infection. Following uptake (up to $4 \mathrm{~h}$ ), the number of c.f.u. recovered from BV2 cells remained constant and was similar among the three strains (Fig. 2). In contrast, quantification of bacterial loads $8 \mathrm{~h}$ after infection demonstrated that intracellular levels of the $\Delta$ sodA mutant were approximately fourfold lower than those of the parental and complemented $\Delta \operatorname{sod} A$ strains $(P<0.05)$ (Fig. 2). By assuming that the growth behaviours of JH22 and the $\Delta$ sodA mutant in the medium growing BV2 cells were essentially identical (data not shown), these differences could presumably be attributed to the lack of intracellular survival of the mutant with respect to the

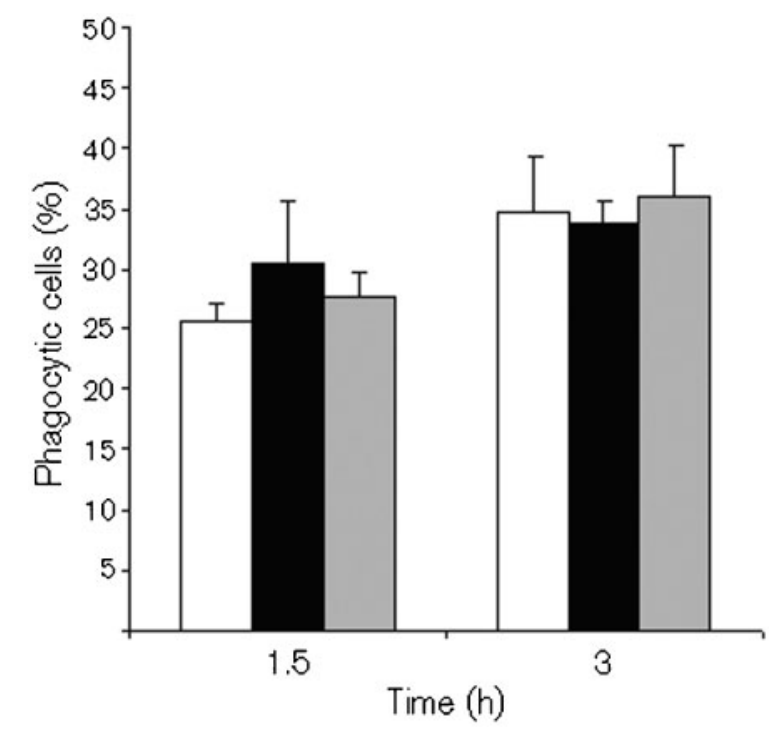

Fig. 1. Phagocytic activity of microglial cells towards $\mathrm{JH} 2-2$ (white bars), the $\triangle$ sodA mutant (black bars) and the complemented $\Delta$ sodA strain (grey bars). BV2 cells were incubated with DAPIlabelled bacteria for the indicated times. Fluorescence of nonphagocytosed bacteria was quenched via treatment with trypan blue before fluorescence of ingested bacteria was measured. Values represent the means $\pm S D$ of three to five independent experiments. 


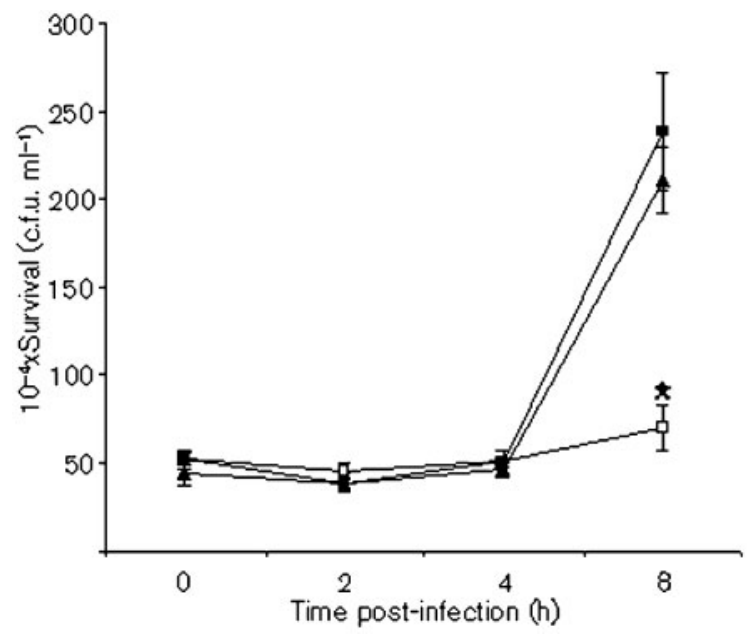

Fig. 2. Survival of $E$. faecalis strains in BV2 cells. Strains $(\boldsymbol{\Delta}, \mathrm{JH} 2-$ 2; $\square, \Delta \operatorname{sod} A$ mutant; $\mathbf{\square}$, complemented $\Delta \operatorname{sod} A$ ) were assayed for survival $0,2,4$ and $8 \mathrm{~h}$ after infection of cells, as described in Methods. Results correspond to the means $\pm S D$ c.f.u. $\mathrm{ml}^{-1}$ from three to five independent experiments. Asterisk indicates a statistically significant difference between JH2-2- and $\Delta$ sodAinfected cells $(P<0.05)$.

parental strain. Similar results were obtained when different host: pathogen ratios were used in the assay (data not shown). These findings suggest that MnSOD confers to E. faecalis resistance against the ROS-mediated killing and digestion by microglia.

\section{MnSOD delays the maturation of $E$. faecalis- containing phagosomes within microglia}

It is known that the killing of internalized pathogens is also due to the fusion of phagosomes with lysosomes, which contain degradative enzymes in an acidic environment. To investigate the events following E. faecalis internalization by microglia, the fate of phagosomes with the ingested bacterial strains was evaluated in terms of acidification and expression of endolysosomal markers (Kinchen \& Ravichandran, 2008). The phagosome maturation, through sequential acquisition of different proteins (i.e. the Rab GTPases) followed by fusion with acidic lysosomes, is of great importance to the response to bacterial pathogens.

First, JH2-2, the $\triangle$ sodA mutant and the complemented $\triangle$ sodA strain labelled with DAPI were exposed to BV2 cells and LysoTracker Red DND-99, a marker for phagosome acidification, was added. Representative overlapping pictures of the different fluorescence channels are shown in Fig. 3. A red colour indicates the presence of an acidic organelle, i.e. the phagolysosomes, whereas a blue fluorescence the presence of bacteria. If bacteria were found in acidic organelles, i.e. a red and a blue colour were present in the same site, this resulted in purple fluorescence and was taken as an indicator of successful phagosome maturation. To quantify the phenomenon, we determined the percentage of acidic phagosomes by counting purple phagosomes within the phagocytic cells. At $1.5 \mathrm{~h}$ post-infection, the percentage of $\Delta \operatorname{sod} A$ mutant-harbouring phagosomes that showed an accumulation of the acid-specific fluorescent dye was significantly higher than that of JH2-2- or complemented $\Delta$ sodA-containing phagosomes (Fig. 4). Similar results were observed at $3 \mathrm{~h}$ post-infection (Fig. 4).

Finally, BV2 cells infected with the $\Delta$ sodA mutant and JH22 strains were quantitatively analysed for the appearance of endolysosomal markers, i.e. Rab5, Rab7, Rab9 and LAMP2. The $\Delta \operatorname{sod} A$ mutant and JH2-2 showed a different degree of colocalization with most of the antibody-stained compartments (Fig. 5). At 30 min post-infection, Rab5-positive phagosomes, and even more Rab7-, Rab9- and LAMP-2positive phagosomes, were present in significantly higher numbers in the $\Delta \operatorname{sod} A$ - than in JH2-2-infected BV2 cells. At $45 \mathrm{~min}$ post-infection, the $\Delta$ sodA-containing phagosomes retained a higher percentage of staining, in terms of endosomal markers, yet such differences were not statistically significant (Fig. 5).

\section{DISCUSSION}

As part of the innate control system of brain infection, microglia encompassing up to $15 \%$ of brain cells are located within the brain parenchyma, just behind the blood-brain barrier, and constitute the main phagocytic population of the central nervous system (Rock et al., 2004; Hauwel et al., 2005). Usually recognized as resident 'amateur' macrophages, they are responsible for the early control of infections as well as for the recruitment of cells of the adaptive immune system required for pathogen clearance (Neglia et al., 2006; Lehnardt, 2010). Like 'professional' macrophages, microglial cells promptly respond to invading micro-organisms through their uptake and internalization into phagosomes, in which they face a hostile environment, characterized by not only a low $\mathrm{pH}$ but also the presence of a number of toxic compounds, e.g. ROS, defensins and lysosomal hydrolases, all potentially contributing to killing and digestion of ingested pathogens (Desjardins et al., 2005).

At present, little is known regarding the molecular mechanisms used by E. faecalis to persist within eukaryotic cells or how the bacterium specifically evades innate and acquired host immune defences (Gilmore et al., 2002). The presence of MnSOD confers to the wild-type strain JH2-2 strong resistance to early killing by BV2 cells, which was indeed significantly decreased in the $\Delta \operatorname{sod} A$ mutant. The similarity of results between the present and other studies (Orsi et al., 2009; Peppoloni et al., 2010) implies that in microglial cells, as well as in 'professional' macrophages, phagocytosis and intracellular killing are not strictly related phenomena, even though the biological function of MnSOD was sufficient for us to rule out any involvement of MnSOD in the process of taking up by BV2 cells. 


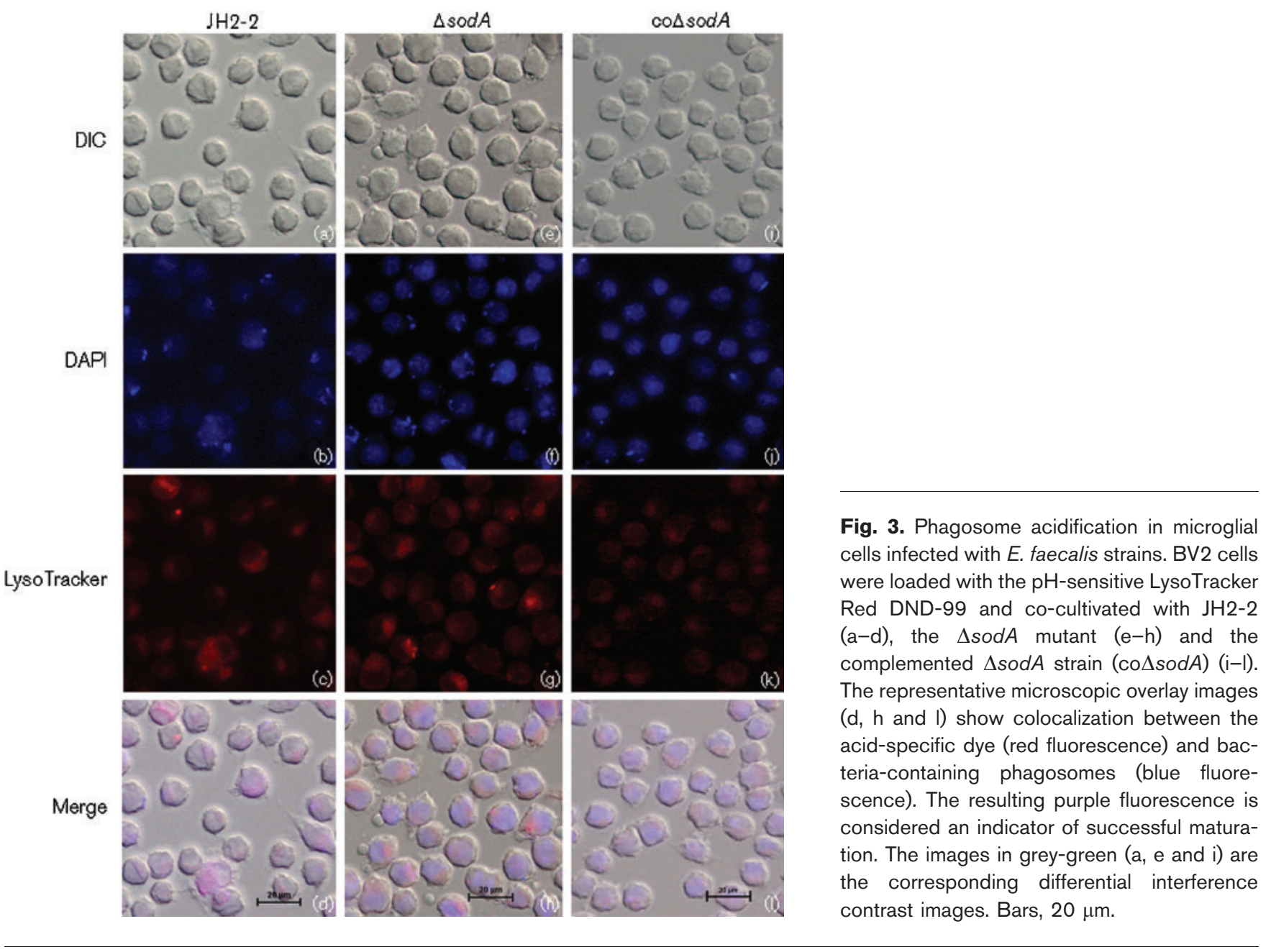

Killing of internalized pathogens is attributed to not only the release of reactive oxygen and nitrogen species but also the fusion of phagosomes with lysosomes, as mentioned above. This process, called phagolysosome biogenesis or phagosome maturation, implies that, after their formation, phagosomes undergo a series of changes, by sequentially fusing with endocytic organelles, such as early endosomes, late endosomes and lysosomes, and by modulating the sequential appearance and disappearance of key phagosome proteins (Desjardins, 1995). A long-standing paradigm is that ROS formed during phagocytosis are primarily inside the phagolysosome, and this fits their definition for being intracellular, i.e. they are detected in the presence of extracellular scavengers (Bylund et al., 2010). Thus, while it is clear that phagolysosome ROS are imperative for effective killing of microbes, our data argue that their removal by the intracellular scavenger activity of MnSOD should result in enhanced survival of the bacterium.

In addition, we examined differences in the fate of $E$. faecalis $\mathrm{JH} 2-2$ phagosomes compared with the $\Delta$ sodA mutant strain to determine whether MnSOD could somehow alter phagolysosome biogenesis. As acidification is one of the major steps of phagosome maturation, we first analysed the percentage of acidic phagosomes bearing both E. faecalis strains. Interestingly, we demonstrated that the deletion of $\operatorname{sod} A$ enhances the acidification process, such that only $\sim 5 \%$ of phagosomes harbouring JH2-2 were fully acidified, with respect to $\sim 20 \%$ seen with the $\Delta \operatorname{sodA}$ mutant. Next, we analysed the pathway for phagosome maturation in terms of recruitment of endolysosomal markers and we demonstrated that the early endosome marker Rab5 was detectable in $>24 \%$ and $9 \%$ of the microglia infected with the $\triangle$ sodA mutant and JH2-2 strains, respectively. More striking differences between the two strains were observed with Rab7, which is known to traffic from early to late endosome or lysosome, and with the late endosome marker Rab9 (Kinchen \& Ravichandran, 2008). The increased maturation of phagosomes housing the $\Delta \operatorname{sod} A$ mutant has been further confirmed by the finding that they were labelled for the LAMP2 marker, a glycoprotein that is specifically localized to acidic lysosome structures (Eskelinen et al., 2003), to an extent that exceeded that seen with the parental strain JH2-2. Although LAMP proteins are required for fusion of lysosomes with phagosomes (Huynh et al., 2007), the precise molecular functions of these proteins remain to be determined (Kinchen \& Ravichandran, 2008). However, 


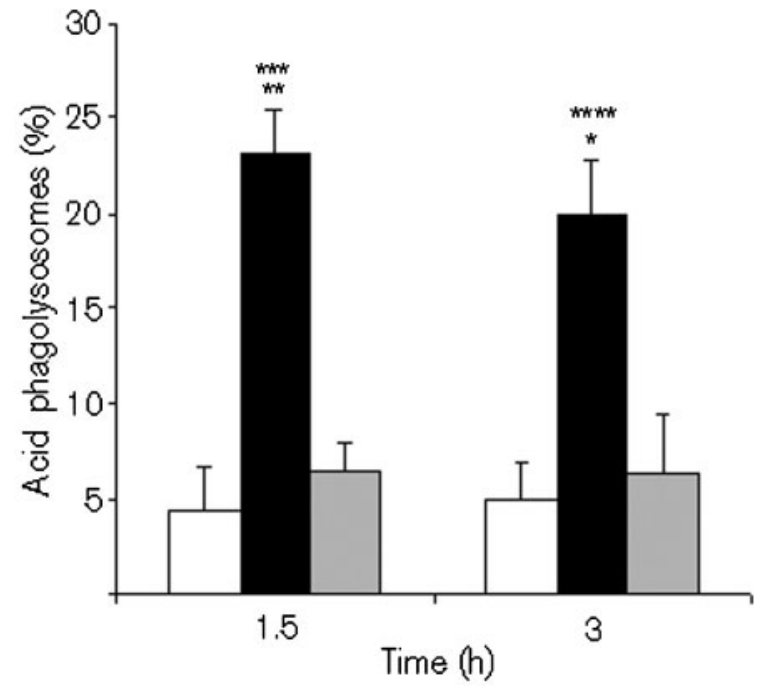

Fig. 4. Quantification of acidic phagosomes in microglial cells infected with $E$. faecalis strains. A suspension of DAPI-labelled $\mathrm{JH} 2-2$ (white bars), the $\Delta \operatorname{sod} A$ mutant (black bars) or the complemented $\triangle$ sodA strain (grey bars) and LysoTracker Red DND-99 was added to BV2 cells and incubated for the indicated time points. Accumulation of the acid-specific dye in phagosomes containing DAPI-labelled bacteria was observed using epifluorescence microscopy. Data represent the percentage of successfully acidified phagosomes with respect to the total number of phagocytic cells counted (a minimum of 200 BV2 cells per bacterial strain), and are the means $\pm S D$ of three to five independent experiments. Asterisks indicate statistically significant differences $\left({ }^{\star} P<0.05, \mathrm{JH} 2-2\right.$ versus $\Delta$ sod $A$ mutant; ${ }^{\star \star} P<0.01$, $\mathrm{JH} 2-2$ versus $\Delta$ sod $A$ mutant; ${ }^{* * *} P<0.01, \Delta$ sod $A$ mutant versus the complemented $\Delta$ sod $A$ strain; ${ }^{* \star * *} P<0.05, \Delta$ sod $A$ mutant versus the complemented $\Delta \operatorname{sod} A$ strain).

defects in LAMP2 by blocking phagosome maturation have been linked to decreased neutrophil-mediated bacterial killing (Beertsen et al., 2008).

Remarkably, our results indicate that the wild-type strain $\mathrm{JH} 2-2$ is more effective than the $\Delta$ sodA mutant in preventing its targeting to compartments displaying late endosomal/lysosomal features and this could impair the bacterium's digestion by microglia to a limited extent and thus favour its survival in the phagocytic cells. A similar effect could also be observed in neutrophils, which are an essential component of the human innate immune system, thus contributing to the pathogenesis of E. faecalis. Further investigations are also required to assess the significance of this finding through elucidation of the intracellular mechanisms triggered by MnSOD or by accumulation of $\mathrm{O}_{2}^{-\cdot}$ to critical levels. An unresolved question is whether ROS directly react with and kill microbes or if they are indirect mediators of killing (Kinchen \& Ravichandran, 2008). Recent evidence suggests that SodA is central to the intrinsic ability of $E$. faecalis to withstand drug-induced killing and $\mathrm{O}_{2}^{-}$is the key effector of bacterial death (Bizzini

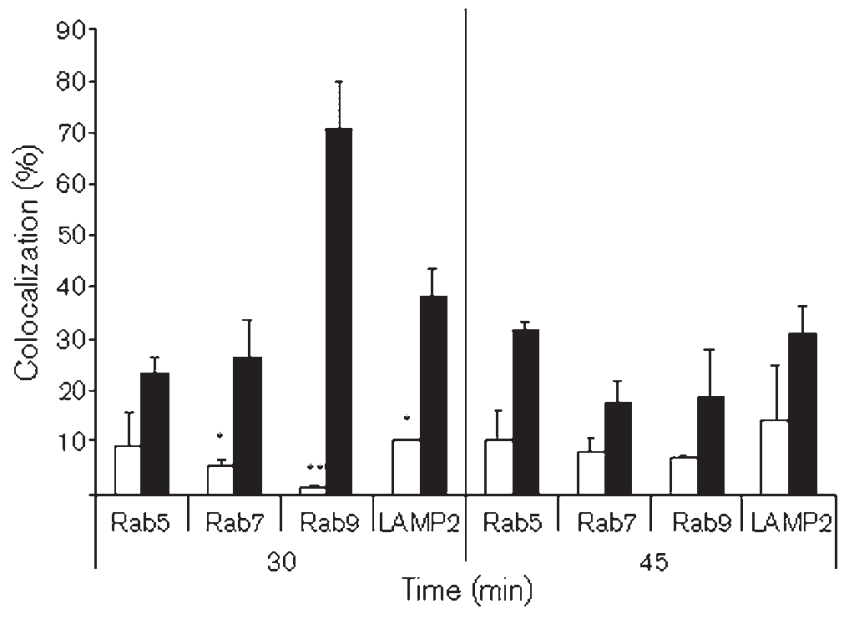

Fig. 5. Assessment of endolysosomal markers in microglial cells infected with $E$. faecalis strains. BV2 cells were co-cultivated with DAPI-labelled JH2-2 (white bars) or the $\Delta$ sodA mutant (black bars) and then incubated with anti-Rab5, -Rab7, -Rab9 or LAMP2 antibodies at the indicated time points. Upon addition of a Chromeo 642-stained secondary antibody, the percentage of colocalization between the antibody-specific dye (red fluorescence) and bacteria-containing phagosomes (blue fluorescence) was determined with respect to the total number counted (at least 200 phagosomes per bacterial strain). Data are based on the mean \pm SD of three independent experiments. Asterisks indicate statistically significant differences $\left({ }^{\star} P<0.05\right.$ and $\left.{ }^{* *} P<0.01\right)$.

et al., 2009). Thus, the identification of the $\mathrm{O}_{2}^{-\cdot}$-sensitive targets or specific superoxide dismutase inhibitors may provide novel insights into the molecular mechanisms of $E$. faecalis death and novel potential targets for antimicrobial intervention.

In conclusion, we have shown for the first time to our knowledge that MnSOD significantly affects the ability of $E$. faecalis to cope with the oxidative damage in microglia, as the mutant defective in this enzyme exhibited marked susceptibility to macrophages in vitro. However, the contribution of oxygen-dependent microbicidal activity against E. faecalis in microglia studied outside the native context of host organ, i.e. the brain, may differ from that occurring in vivo. Studies using a mouse model of E. faecalis meningitis will be undertaken to test this hypothesis.

\section{ACKNOWLEDGEMENTS}

We wish to acknowledge $\mathrm{Mr}$ Antonio Martino for excellent informatic support. Very special thanks are also given to Dr Andrea Ardizzoni for helpful suggestions and a critical review of the manuscript.

\section{REFERENCES}

Beertsen, W., Willenborg, M., Everts, V., Zirogianni, A., Podschun, R., Schröder, B., Eskelinen, E. L. \& Saftig, P. (2008). Impaired 
phagosomal maturation in neutrophils leads to periodontitis in lysosomal-associated membrane protein-2 knockout mice. J Immunol 180, 475-482.

Bizzini, A., Zhao, C., Auffray, Y. \& Hartke, A. (2009). The Enterococcus faecalis superoxide dismutase is essential for its tolerance to vancomycin and penicillin. J Antimicrob Chemother 64, 1196-1202.

Blasi, E., Barluzzi, R., Bocchini, V., Mazzolla, R. \& Bistoni, F. (1990). Immortalization of murine microglial cells by a v-raf/v-myc carrying retrovirus. J Neuroimmunol 27, 229-237.

Brett, P. J., Burtnick, M. N., Su, H., Nair, V. \& Gherardini, F. C. (2008). iNOS activity is critical for the clearance of Burkholderia mallei from infected RAW 264.7 murine macrophages. Cell Microbiol 10, 487-498.

Bylund, J., Brown, K. L., Movitz, C., Dahlgren, C. \& Karlsson, A. (2010). Intracellular generation of superoxide by the phagocyte NADPH oxidase: how, where, and what for? Free Radic Biol Med 49, 1834-1845.

CLSI (2010). Performance standards for antimicrobial susceptibility testing. CLSI document M100-S20. 20th informational supplement. Wayne, PA: Clinical and Laboratory Standards Institute.

Desjardins, M. (1995). Biogenesis of phagolysosomes: the 'kiss and run' hypothesis. Trends Cell Biol 5, 183-186.

Desjardins, M., Houde, M. \& Gagnon, E. (2005). Phagocytosis: the convoluted way from nutrition to adaptive immunity. Immunol Rev 207, 158-165.

Eskelinen, E. L., Tanaka, Y. \& Saftig, P. (2003). At the acidic edge: emerging functions for lysosomal membrane proteins. Trends Cell Biol 13, 137-145.

Fernández Guerrero, M. L., Goyenechea, A., Verdejo, C., Roblas, R. F. \& de Górgolas, M. (2007). Enterococcal endocarditis on native and prosthetic valves: a review of clinical and prognostic factors with emphasis on hospital-acquired infections as a major determinant of outcome. Medicine (Baltimore) 86, 363-377.

Gilmore, M. S., Coburn, P. S., Nallapareddy, S. R. \& Murray, B. E. (2002). Enterococcus virulence. In The Enterococci: Phathogenesis, Molecular Biology, Antibiotic Resistance and Infection Control, pp. 301354. Edited by M. S. Gilmore. Washington, DC: American Society for Microbiology.

Hauwel, M., Furon, E., Canova, C., Griffiths, M., Neal, J. \& Gasque, P. (2005). Innate (inherent) control of brain infection, brain inflammation and brain repair: the role of microglia, astrocytes, "protective" glial stem cells and stromal ependymal cells. Brain Res Rev 48, 220-233.

Huycke, M. M., Sahm, D. F. \& Gilmore, M. S. (1998). Multiple-drug resistant enterococci: the nature of the problem and an agenda for the future. Emerg Infect Dis 4, 239-249.

Huynh, K. K., Eskelinen, E. L., Scott, C. C., Malevanets, A., Saftig, P. \& Grinstein, S. (2007). LAMP proteins are required for fusion of lysosomes with phagosomes. EMBO J 26, 313-324.
Imlay, J. A. (2003). Pathways of oxidative damage. Annu Rev Microbiol 57, 395-418.

Kinchen, J. M. \& Ravichandran, K. S. (2008). Phagosome maturation: going through the acid test. Nat Rev Mol Cell Biol 9, 781-795.

Lehnardt, S. (2010). Innate immunity and neuroinflammation in the CNS: the role of microglia in Toll-like receptor-mediated neuronal injury. Glia 58, 253-263.

Murray, B. E. (2000). Vancomycin-resistant enterococcal infections. $N$ Engl J Med 342, 710-721.

Neglia, R., Colombari, B., Peppoloni, S., Orsi, C., Tavanti, A., Senesi, S. \& Blasi, E. (2006). Adaptive response of microglial cells to in vitro infection by Candida albicans isolates with different genomic backgrounds. Microb Pathog 41, 251-256.

Orsi, C. F., Colombari, B., Ardizzoni, A., Peppoloni, S., Neglia, R., Posteraro, B., Morace, G., Fadda, G. \& Blasi, E. (2009). The ABC transporter-encoding gene AFR1 affects the resistance of Cryptococcus neoformans to microglia-mediated antifungal activity by delaying phagosomal maturation. FEMS Yeast Res 9, 301-310.

Peppoloni, S., Ricci, S., Orsi, C. F., Colombari, B., De Santi, M. M., Messinò, M., Fabio, G., Zanardi, A., Righi, E. \& other authors (2010). The encapsulated strain TIGR4 of Streptococcus pneumoniae is phagocytosed but is resistant to intracellular killing by mouse microglia. Microbes Infect 12, 990-1001.

Pintado, V., Cabellos, C., Moreno, S., Meseguer, M. A., Ayats, J. \& Viladrich, P. F. (2003). Enterococcal meningitis: a clinical study of 39 cases and review of the literature. Medicine (Baltimore) 82, 346364.

Rock, R. B., Gekker, G., Hu, S., Sheng, W. S., Cheeran, M., Lokensgard, J. R. \& Peterson, P. K. (2004). Role of microglia in central nervous system infections. Clin Microbiol Rev 17, 942-964.

Sarkar, S., Bhagat, I., DeCristofaro, J. D., Wiswell, T. E. \& Spitzer, A. R. (2006). A study of the role of multiple site blood cultures in the evaluation of neonatal sepsis. J Perinatol 26, 18-22.

Suppli, M., Aabenhus, R., Harboe, Z. B., Andersen, L. P., Tvede, M. \& Jensen, J. U. (2010). Mortality in enterococcal bloodstream infections increases with inappropriate antimicrobial therapy. Clin Microbiol Infect. (Epub ahead of print).

Verneuil, N., Mazé, A., Sanguinetti, M., Laplace, J. M., Benachour, A., Auffray, Y., Giard, J. C. \& Hartke, A. (2006). Implication of (Mn)superoxide dismutase of Enterococcus faecalis in oxidative stress responses and survival inside macrophages. Microbiology 152, 25792589.

Willems, R. J. \& Bonten, M. J. (2007). Glycopeptide-resistant enterococci: deciphering virulence, resistance and epidemicity. Curr Opin Infect Dis 20, 384-390.

Edited by: P. C. F. Oyston 